\title{
Ricerche su Ostia e il suo territorio
}

Il volume raccoglie gli Atti del Terzo Seminario Ostiense, tavola rotonda internazionale ormai divenuta annuale presso l'Ecole française de Rome, articolata, come nei seminari precedenti, in due sezioni. La prima, tematica, ha come oggetto Ostia e la riva destra del Tevere, e si apre con contributi che vanno dall'età protostorica (Bronzo recente e finge) all'età arcaica. Per il periodo romano, accanto alla presentazione di scan (1) perie di materiali, deterninanti ai fini fine degli anni ' 80 del secolo sconso. La seconda sezione aceogre contibei quali approfondiscono aspetti del vastissimo, e ancora solo parzialmente esplorato, patrimonio epigrafico ostiense, nonché i risultati di scavi e di sondaggi stratigrafici recenti nel contesto della città, interessanti anche per chiarire meglio l'attitudine e il programma operativo degli archeologi al tempo del Grande Scavo del 1938-42.

Mireille Cébeillac-Gervasoni, già membro dell'École française de Rome, è stata direttrice del Centre Jean Bérard di Napoli e, successivamente, direttrice di ricerca al CNRS (UMR 8210, ANHIMA). I suoi lavori hanno riguardato in grafia e la storia di Ostia antica. È stata autrice, insieme a Maria Letizia Caldelli e Fausto Zevi, del volume Ostia. Cento iscrizioni in contesto (Roma, 2010), e una delle promotrici dei «Seminari Ostiensi» all'École française de Rome.

Nicolas Laubry, già allievo dell'École normale supérieure (Parigi) e membro dell'École française de Rome, maître de conférences in storia romana all'Università di Paris-Est Créteil, è attualmente direttore degli studi per l'Antichità presso l'Ecole française de Rome. Tra i suoi principali interessi vi sono l'epigrafia dell' Occidente romano (Italia, Gallia) e la storia religiosa, istituzionale e sociale delle pratiche funerarie tra la fine della Repubblica e gli inizi della Tarda Antichità.

Fausto Zevi, perfezionatosi in Archeologia classica fra Roma e Atene, per quasi 25 anni ha lavorato nelle Soprintendenze archeologiche, dapprima a Ostia, poi come Soprintendente a Napoli e Pompei e a Roma; in seguito ha avuto la cattedra di archeologia nelle Università di Napoli e poi di Sapienza Università di Roma. Tra le sue numerose pubblicazioni su vari aspetti dell'Italia antica, molte sono state dedicate a Ostia. E accademico dei Lincei.

\section{Ricerche su Ostia e il suo territorio}

a cura di Mireille Cébeillac-Gervasoni $\dagger$, Nicolas LaUbry e Fausto ZeVI
P 


\section{RICERCHE SU OSTIA E IL SUO TERRITORIO}



C OLLECTION DE L'ÉCOLL FRANÇAISE DE ROME 553

\section{RICERCHE SU OSTIA E IL SUO TERRITORIO}

ATTI DEL TERZO SEMINARIO OSTIENSE

(ROMA, ÉCOLE FRANÇAISE DE ROME, 21-22 OTTOBRE 2015)

a cura di Mireille Cébeillac-Gervasoni $\dagger$

Nicolas Laubry e Fausto ZeVI 
Ricerche su Ostia e il suo territorio : atti del terzo seminario ostiense (Roma, École française de Rome, 21-22 ottobre 2015) / a cura di Mireille Cébeillac-Gervasoni, Nicolas Laubry e Fausto Zevi

Rome : École française de Rome, 2019

(Collection de l'École française de Rome, 0223-5099 ; 553)

ISBN 978-2-7283-1332-7 (br.)

ISBN 978-2-7283-1333-4 (EPub)

Disponible sur Internet : <http://books.openedition.org/efr/3637> @ 2018

DOI : 10.4000/books.efr.3637

1. Fouilles archéologiques -- Italie -- Ostie (ville ancienne) -- Actes de congrès 2. Urbanisme -- Italie -- Ostie (ville ancienne) -- Actes de congrès 3. Tombes -- Italie -- Isola Sacra (Italie) -- Antiquité -- Actes de congrès

4. Inscriptions latines -- Italie -- Ostie (ville ancienne) -- Actes de congrès 5. Ostie (ville ancienne) -- Antiquité -- Actes de congrès

I. Cébeillac-Gervasoni, Mireille (1942-2017) II. Laubry, Nicolas, 1980-

III. Zevi, Fausto IV. École française de Rome. Seminario ostiense

(3 ; 2015 ; Rome, Italie)

CIP - Bibliothèque de l'École française de Rome

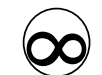

ISO/CD 9706

(C) - École française de Rome - 2019

ISSN 0223-5099

ISBN 978-2-7283-1332-7 


\section{MIREILLE CÉBEILLAC-GERVASONI †, NICOLAS LAUBRY E FAUSTO ZEVI}

\section{PREMESSA}

Presentiamo gli atti della tavola rotonda internazionale «Terzo Seminario Ostiense» svoltasi il 22-23 ottobre 2015 presso la sede dell'EFR di Piazza Navona, che vedono la luce nella prestigiosa Collection de l'École française de Rome. L'incontro ha ottenuto un notevole successo presso il pubblico, non solo italiano, sempre desideroso di conoscere i dati inediti presentati durante questi incontri e di partecipare alle discussioni. I colleghi archeologi, epigrafisti e storici, italiani e stranieri, che lavorano ad Ostia o su tematiche ostiensi, hanno risposto positivamente e hanno presentato $\mathrm{i}$ loro contributi nel corso di queste due intense giornate. Questo mette in evidenza quanto fosse necessaria la ripresa degli incontri annuali dedicati a Ostia, come già avevano mostrato i precedenti colloqui, pubblicati nei MEFRA degli scorsi anni.

Secondo una prassi ormai consolidata, l'incontro si è articolato in due sezioni principali, con una prima giornata riservata a problematiche ostiensi puntuali e mirate, dedicata quest'anno alla riva destra del Tevere, con un'attenzione particolare alla necropoli di Porto all'Isola Sacra, che alla fine degli anni '80 era stata oggetto di importanti campagne di scavo solo parzialmente pubblicate - una lacuna che l'edizione di questi atti viene in parte a colmare. $\mathrm{La}$ seconda giornata di "Varia» era destinata alla presentazione di scavi e studi inediti ; fedele alla tradizione, questa sezione ha permesso di conoscere lavori in corso o appena conclusi ad Ostia da parte di archeologi, epigrafisti e storici. Gli antropologi del Museo Pigorini sono intervenuti con l'analisi di resti umani rinvenuti in tombe scoperte negli scavi della primavera del 2015 , in un confronto ricco di integrazioni e conferme con i risultati conseguiti dagli archeologi e dagli epigrafisti.

Ringraziamo quanti ci hanno permesso di organizzare e svolgere queste giornate, la Soprintendenza archeologica competente e le responsabili scientifiche di Ostia Antica, Cinzia Morelli e Paola Germoni, per la loro adesione immediata al progetto e l'aiuto costante per la buona riuscita dell'incontro. All'École française de Rome e alla sua direttrice Catherine Virlouvet siamo grati per l'ospitalità nella sede di piazza Navona nelle due giornate dell'incontro, l'accoglienza e il soggiorno dei relatori, i pranzi per tutti i relatori ed i contributi per il vitto per quanti non risiedevano a Roma. Un grazie particolare a Stéphane Bourdin, directeur des études della sezione Antiquité dell'EFR, per aver sostenuto e seguito i nostri progetti e per aver inoltre accettato di pubblicare gli atti nella collana dell'EFR ; a Giulia Cirenei, segretaria della sezione antichità dell'EFR, che ha collaborato attivamente ad un'organizzazione impeccabile dell'incontro e del soggiorno degli ospiti stranieri. 



\section{LISTA DELLE ABBREVIAZIONI}

Le riviste sono abbreviate secondo l'Archäologische Bibliographie e gli autori e opere delle fonti classiche secondo l'Oxford Classical Dictionnary (V ed.). Inoltre, sono state usate le abbreviazioni seguenti :

$A E=$ L'Année épigraphique

ANRW = Aufstieg und Niedergang der Römischen Welt

$B M C=$ British Museum Catalogue

CIG $=$ Corpus Inscriptionum Graecarum

$C I L=$ Corpus Inscriptionum Latinarum

EDCS = Epigraphische Datenbank Clauss/Slaby

$I C U R=$ Inscriptiones Christianae Urbis Romae

$I G U R=$ Inscriptiones Graecae Urbis Romae

$I G=$ Inscriptiones Graecae

$I G L S=$ Inscriptions grecques et latines de la Syrie

ILAlg = Inscriptions latines de l'Algérie

$I L S$ = Inscriptiones Latinae Selectae

InscrIt = Inscriptiones Italiae

IScM = Inscriptiones Scythiae Minoris

LIMC = Lexicon Iconographicon Mythologiae Classicae

$L S O=$ Lateres Signati Ostienses

LTURS = Lexicon Topographicum Urbis Romae, Suburbium

PAnt $=$ The Antinoopolis Papyri

$P G M=$ Papyri Graecae Magicae

PLRE = Prosopography of the Later Roman Empire

$R E=$ Realencyclopädie der klassischen Altertumswissenschaft

$R I C=$ Roman Imperial Coinage

$R P C=$ Roman Provincial Coinage

SEG = Supplementum Epigraphicum Graecum

Suppl. Mag. = Supplementum Magicum

TAM = Tituli Asiae Minoris

ThesCRA = Thesaurus cultus et rituum antiquorum 


\section{EMANUELA BORGIA}

\section{UNA TABELLA DEFIXIONIS DALLA NECROPOLI DELL'ISOLA SACRA}

Una tabella defixionis plumbea è stata rinvenuta negli scavi condotti nella necropoli dell'Isola Sacra negli anni 1988-1989, nel saggio n. 25 (fig. 1). La laminetta era ancora arrotolata ed è stata in un certo qual modo riscoperta, restaurata e analizzata preliminarmente negli anni 1999-2000, in occasione dello studio sistematico dei materiali provenienti dagli scavi, condotto sotto gli auspici della allora Soprintendenza per i Beni Archeologici di Ostia ${ }^{1}$.

Prima di prendere in esame in dettaglio il testo del documento è opportuno esaminarne il contesto di rinvenimento, in questo caso ben noto ed indagato stratigraficamente. Ciò può contribuire, pur se solo parzialmente, all'am-

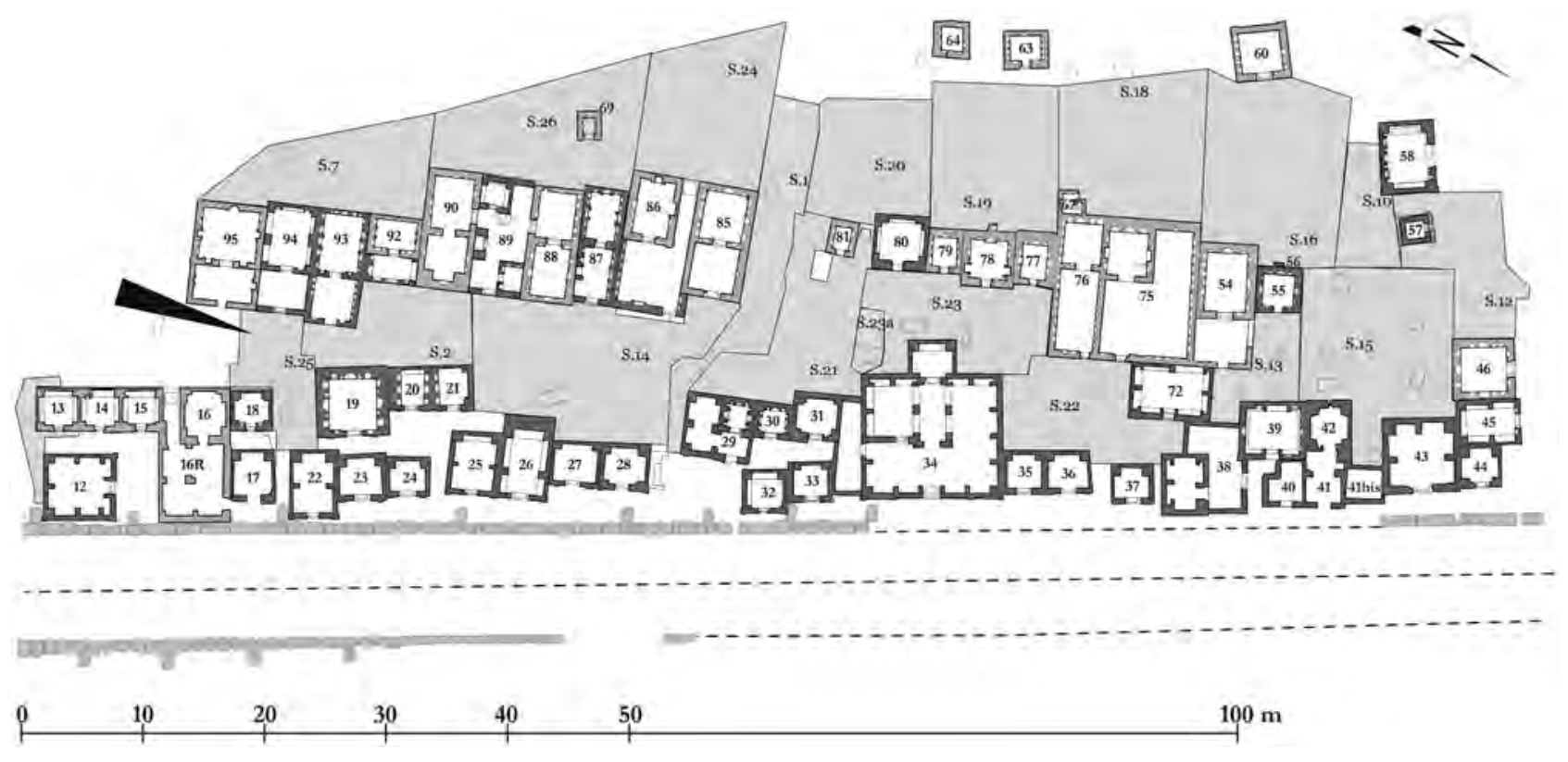

Fig. 1 - Necropoli dell'Isola Sacra: planimetria generale con localizzazione del saggio 25 .

\footnotetext{
${ }^{1}$ Ringrazio sentitamente la dott.ssa A. Gallina Zevi, allora Soprintendente per i Beni Archeologici di Ostia, per avermi affidato lo studio di questo documento e le dott. sse J. E. Shepherd e P. Germoni per la grande disponibilità dimostratami durante le diverse fasi dello studio del documento. La laminetta è stata srotolata e restaurata da
}

I. Reindell ed è oggi conservata nei Nuovi Depositi di Ostia Antica (N. inv. 55832), Soprintendenza Speciale per il Colosseo, il Museo Nazionale Romano e l'Area Archeologica di Roma, Sede di Ostia Antica. Un ringraziamento particolare va a G. Bevilacqua per i preziosi consigli e suggerimenti fornitimi durante la stesura di questo contributo. 
pliamento delle conoscenze relative ai riti magici legati alla deposizione delle defixiones. Secondo una consuetudine ampiamente diffusa nelle pratiche magiche, la defixio fu deposta in un'area funeraria, dove si riteneva che più facilmente il messaggio di malaugurio venisse ricevuto dalle divinità infere, anche grazie all'intermediazione dell'anima del defunto considerato un tramite eccellente con l'aldilà ${ }^{2}$.

La laminetta era arrotolata e inserita all'interno di un'anfora che costituiva il segnacolo di una sepoltura, anch'essa in anfora (fig. 2). Il segnacolo, essendo posto in verticale, garantiva in maniera ottimale, secondo le credenze magiche, una comunicazione immediata con l'anima del defunto. L'anfora-segnacolo è la n. 674 ed è pertinente al tipo Dressel $14=$ Beltrán 4A, mentre la sottostante sepoltura (n. 16) era contenuta nell'anfora n. 695, del tipo Tripolitana III (fig. 3-4) ${ }^{3}$. Le indicazioni desu- mibili dallo studio dei reperti scheletrici indicano che il defunto era un individuo infantile di sesso indeterminato (3-4 anni $)^{4}$; sulla clavicola è stato rinvenuto un asse o dupondio di Lucio Vero ${ }^{5}$. Tra i materiali restituiti dal riempimento del segnacolo (US 1307) è da ricordare un incensiere in ceramica comune ${ }^{6}$.

L'analisi del contesto di rinvenimento fornisce un importante terminus post quem per la datazione della laminetta, che fu evidentemente deposta in un'epoca posteriore alla realizzazione della sepoltura, risalente agli anni successivi al 161 d.C. Rimane il problema di definire, se possibile, quanto tempo sia intercorso tra la sepoltura e la sua «manomissione» per l'inserimento della defixio. Il dato stratigrafico non fornisce purtroppo alcuna precisa indicazione in merito, in quanto la situazione relativa alle fasi successive alla sepoltura appariva piuttosto compromessa al momento delle

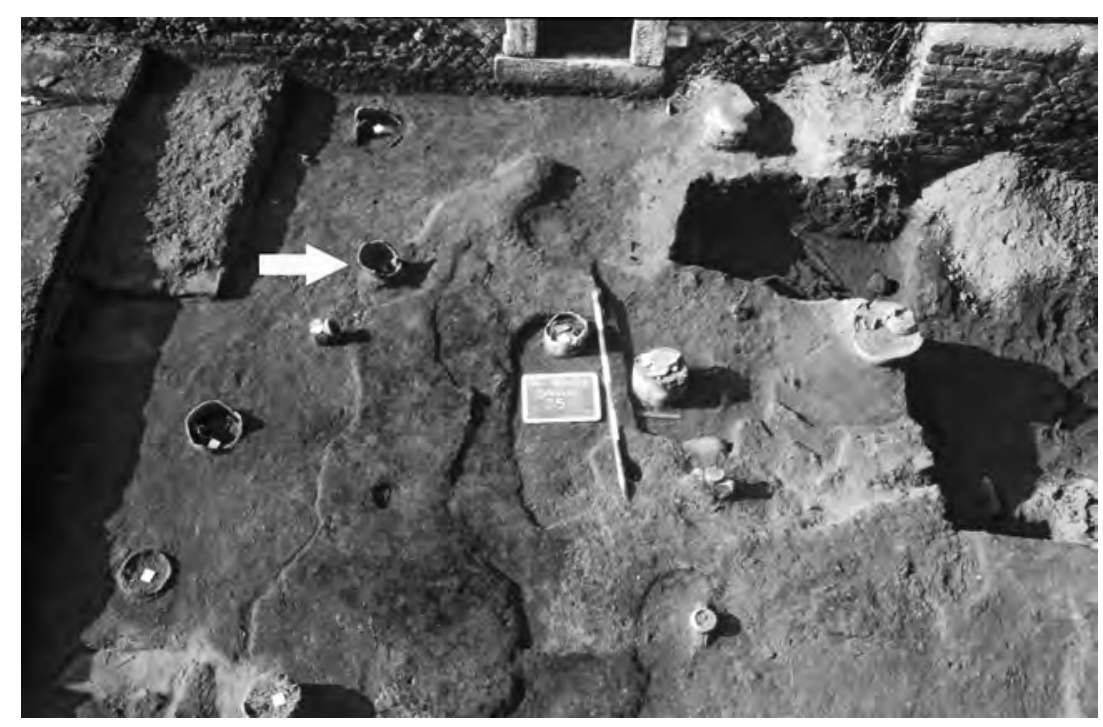

Fig. 2 - Necropoli dell'Isola Sacra: particolare del saggio 25, con indicazione dell'anfora segnacolo (n. 674) che conteneva la tabella defixionis.

2 Si vedano in merito, tra gli altri, Cesano 1910, p. 1587-1590; Youtie - Bonner 1937, p. 45; Panciera 1968, p. 333; Jordan 1985a; Jordan 1985b, p. 152-153; Gager 1992; Graf 1994, p. 148-149; Bevilacqua 2010, p. 23.

3 Le anfore provenienti dagli scavi nella necropoli dell'Isola Sacra sono state studiate da L. Rustico, A. Valchera e R. Zaccagnini, alla cui cortesia devo le informazioni relative alle tipologie anforiche relative alla sepoltura n. 16 del saggio 25. L'anfora Dressel 14 = Beltrán 4A si data tra la metà del I ed il II sec. d.C. con attardamenti fino al III sec. d.C., mentre la Tripolitana III è diffusa tra il II e la seconda metà del III sec. d.C., anch'essa con attardamenti nel IV e addirittura nel V sec. d.C.

${ }^{4}$ Per quanto concerne gli studi antropologici, condotti dal gruppo di ricerca diretto da L. Bondioli del Museo Nazionale Preistorico Etnografico Luigi Pigorini, si vedano infra i contributi.

5 Per i dati numismatici ringrazio F. Taglietti e L. Camilli, che hanno curato lo studio delle monete.

${ }^{6}$ La ceramica comune è stata studiata da C. Coletti e P. Olivanti a cui devo l'indicazione della presenza di tale incensiere, databile genericamente tra il II e il IV sec. d.C. 


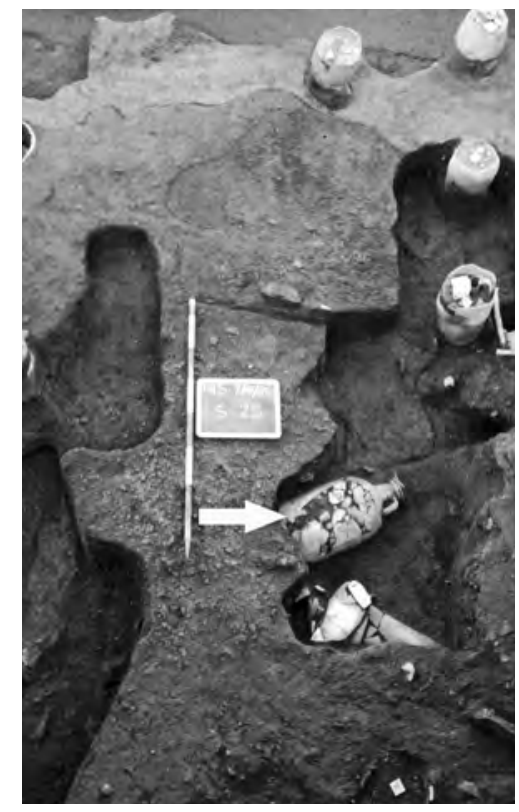

Fig. 3 - Necropoli dell'Isola Sacra: particolare del saggio 25, con indicazione dell'anfora n. 695 contenente la sepoltura.

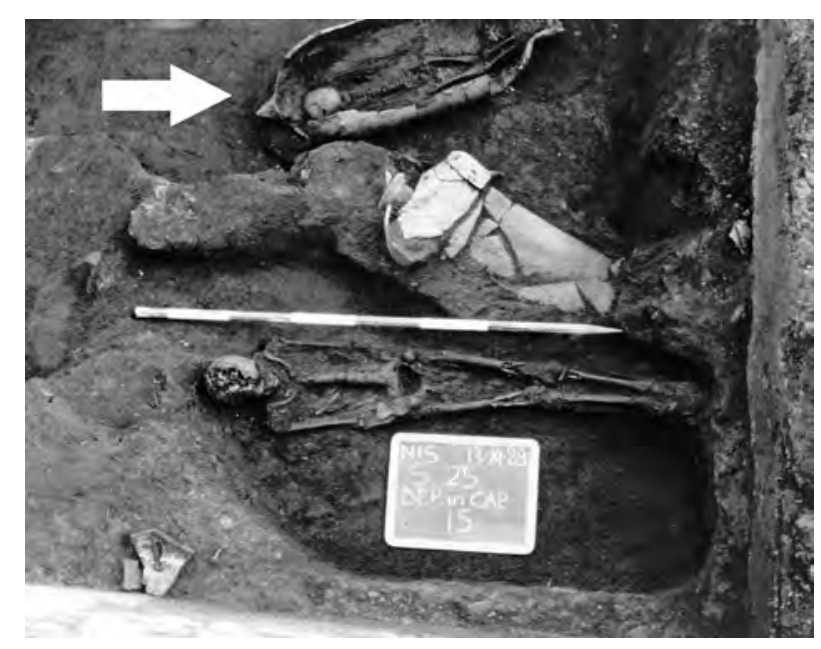

Fig. 4 - Necropoli dell'Isola Sacra: la sepoltura infantile nell'anfora n. 695 (in alto nella foto).

\footnotetext{
${ }^{7}$ Gli scavi condotti dal Calza hanno rimosso indiscriminatamente tutti gli strati superficiali di sabbia (Calza 1940, p. 29-60, in part. p. 32, 44-46, fig. 9-10).

${ }^{8}$ Un confronto piuttosto stringente, sia dal punto di vista del contesto di rinvenimento (e quindi delle pratiche magiche di deposizione della maledizione) sia per quanto concerne la cronologia, si può stabilire con una tabella defixionis latina rinvenuta ad Ostia nella necropoli della Via Ostiense, all'interno della tomba a cappuccina n. 11, databile alla seconda metà del II sec. d.C. Si vedano in merito: per la tomba, Floriani Squarciapino 1958, p. 29-30; per la defixio, Solin 1968, p. 3-5 e 7-10.
}

indagini del 1988-19897. Cionondimeno sembra verosimile supporre che la laminetta sia stata deposta in un'epoca non troppo distante dalla sepoltura stessa, quando la necropoli era ancora almeno parzialmente in uso e di conseguenza l'anfora-segnacolo doveva emergere dal terreno ed essere ben visibile ${ }^{8}$. Tutti gli elementi in nostro possesso inducono dunque a ritenere che la defixio risalga grosso modo agli ultimi anni del II o ai primi decenni del III sec. d.C., cronologia che non viene smentita, come si vedrà, dall'analisi paleografica del testo.

Il fatto che la defixio sia stata deposta proprio in una sepoltura infantile lascia adito alla suggestione che la scelta non sia stata incidentale, visto che, come è noto, le anime degli

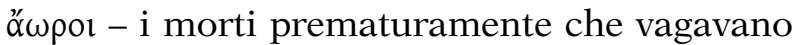
nei cimiteri per un certo periodo prima di giungere nell'aldilà - erano considerate quelle più adatte a trasmettere i messaggi di malaugurio'. Certo ci si chiede come sia possibile che colui che depositò la laminetta nella tomba fosse a conoscenza dell'identità del defunto e soprattutto della sua età, visto che la sepoltura, molto semplice e povera, non ha restituito epigrafi e che l'unico elemento distintivo visibile era l'anfora segnacolo, su cui non si è preservata alcuna iscrizione dipinta né appaiono segni identificativi ${ }^{10}$. Un'eventualità, che non è in alcun modo accertabile ma comunque appare suggestiva, è che il defigens o il suo agente conoscessero l'identità del defunto, nel qual caso il momento della deposizione della defixio si avvicinerebbe di molto, in termini di tempo, alla sepoltura stessa. In ogni caso, in assenza di ulteriori indizi, è forse più prudente lasciare aperta l'ipotesi che la scelta della sepoltura sia da ascrivere a motivi di mera casualità.

\footnotetext{
${ }^{9}$ Se tale interpretazione dovesse rivelarsi esatta, si avrebbe una conferma del fatto che le tombe di giovani fossero predilette per la deposizione delle defixiones, come ipotizzato tra l'altro da Graf, il quale lamenta lo stato inadeguato della documentazione archeologica in merito (Graf 1994, p. 174-175). Su questo problema cf. anche le note di Jordan 1985b, p. 152.

${ }^{10}$ Naturalmente rimane del tutto ignoto se le sepolture a terra fossero in qualche modo identificabili da parte di estranei al gruppo familiare del defunto, magari con evidenze in materiale deperibile non rintracciabile in fase di scavo.
} 
Per quanto concerne il contesto topografico (fig. 5) della sepoltura, esso può aiutarci a delineare, seppure per via congetturale, alcuni aspetti di quella parte pratica del rito magico - e quindi della storia sociale sottesa a tali rituali di cui sempre labili sono le tracce a nostra disposizione $^{11}$. L'ubicazione della sepoltura n. 16 (lasciando in secondo piano la questione se la scelta sia stata casuale o deliberata) era tale da consentire una certa riservatezza per chi, clandestinamente e di certo temendo di essere visto, procedette con la deposizione della laminetta all'interno del segnacolo. Siamo infatti non sulla strada principale della necropoli, ma alle spalle della prima fila di tombe monumentali, sul versante occidentale. Peraltro la tomba in questione si trova subito addossata ad una kline della tomba monumentale 95, nella rientranza formata tra quest'ultima e la facciata della tomba monumentale 94, quindi in posizione relativamente nascosta (fig. 6); allo stesso tempo essa non era però troppo arretrata e quindi piuttosto facilmente raggiungibile da quella che ipotizziamo fosse la stradina o il percorso che correva nell'area, estensivamente occupata da sepolture terragne ${ }^{12}$. Nulla sappiamo in merito al rituale di deposizione, che certamente prevedeva la recitazione di preghiere e formule magiche, spesso ripetitive, non necessariamente riportate sulla laminetta ${ }^{13}$.

Veniamo dunque all'analisi della tabella defixionis (fig. 7-8). La laminetta plumbea ${ }^{14}$,

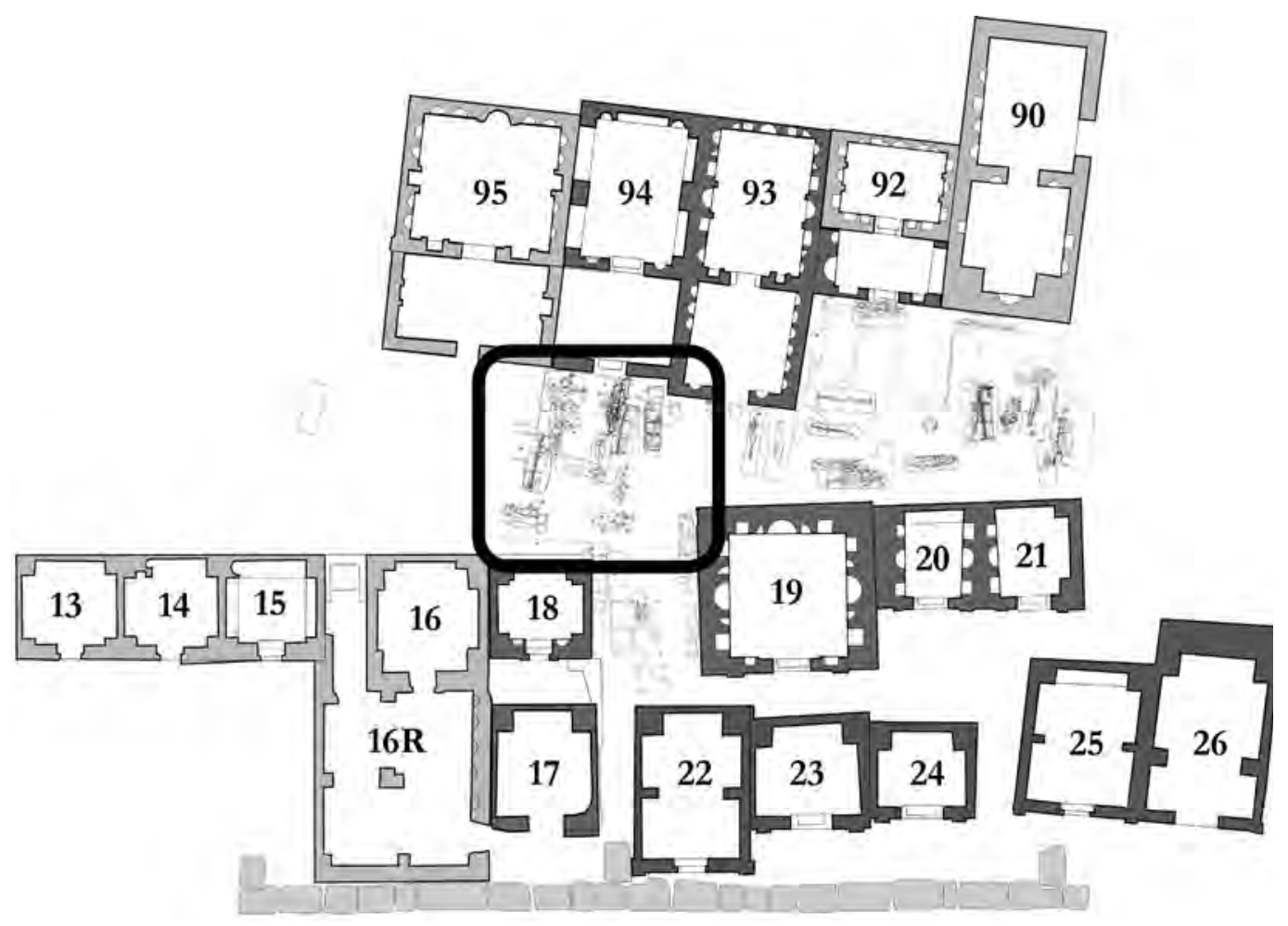

Fig. 5 - Necropoli dell'Isola Sacra: stralcio della planimetria in corrispondenza del saggio 25 .

${ }^{11}$ Per un preliminare studio che propone un approccio di questo genere alla lettura delle defixiones, incentrato sulle province africane, cf. Mura 1996.

${ }^{12}$ Si veda supra il contributo di P. Olivanti e M. Spanu.

${ }^{13}$ Laspetto interessante di questi testi è infatti che, dopo la loro lettura al momento della deposizione, erano destinati a rimanere nascosti e mai più letti; ma il potere del messaggio permaneva, grazie anche al fatto di essere inciso su un materiale durevole. La loro funzione di veicolo di comunicazione veniva affidata alla perpetuazione silenziosa di un incantesimo probabilmente effettuato a voce. Bevilacqua 2010, p. 91; Piccaluga 2010, p. 15-18. Cf. Bravo 1995, p. 520.

${ }^{14}$ Non sono state effettuate analisi di laboratorio che indichino l'esatta composizione chimica del materiale: sappiamo che, oltre al piombo puro, sono frequenti nelle defixiones i casi dell'uso di leghe di piombo, stagno e rame. L'uso del piombo a fini magici viene prescritto anche in un papiro recante dei dettami rituali (PGM LXXVIII, 3; Betz 1986, p. 299). Cf. Bevilacqua 2010, p. 32-36. 


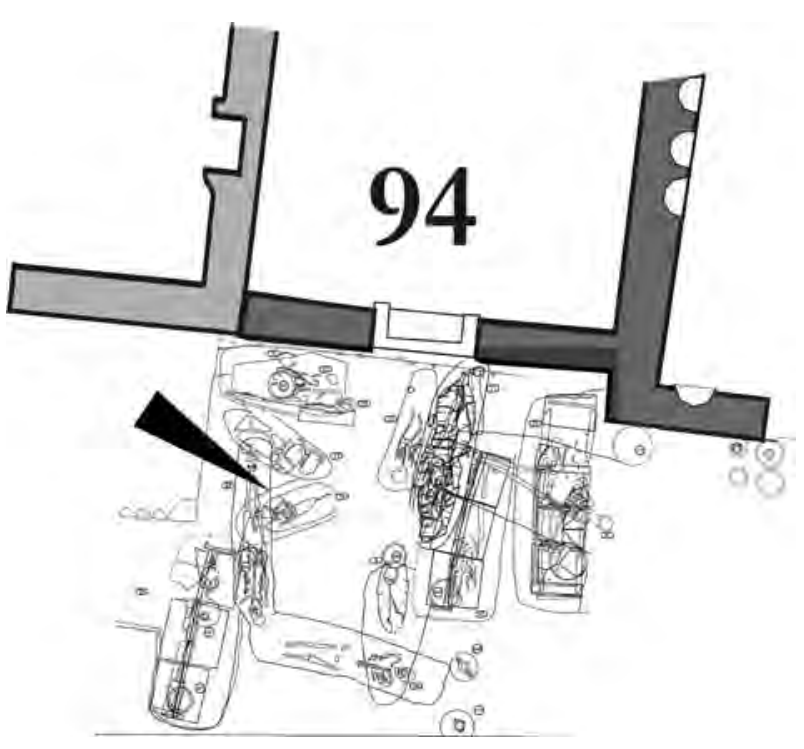

Fig. 6 - Necropoli dell'Isola Sacra: ubicazione della sepoltura in anfora n. 16.

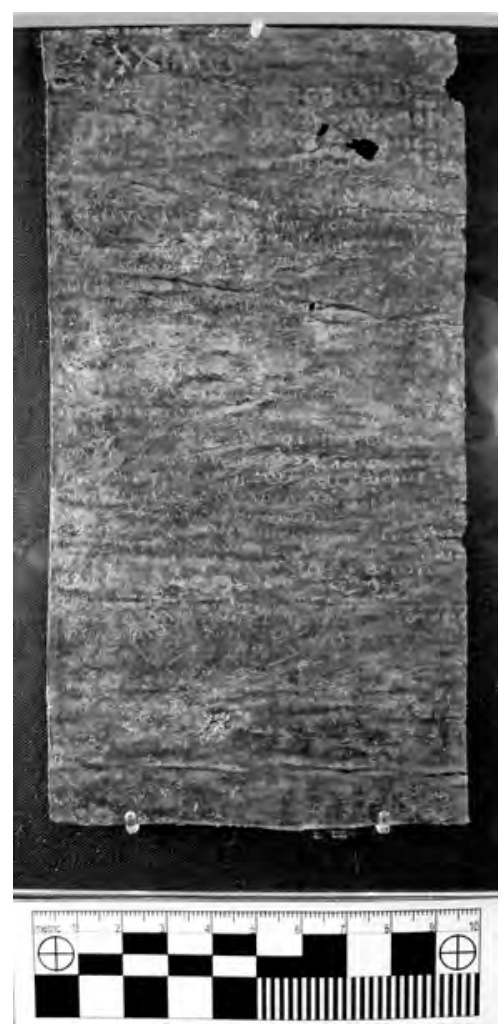

Fig. 7 - La tabella defixionis.

${ }^{15}$ Degno di nota è il fatto che si sia scelto il greco come lingua di redazione della defixio, ad eccezione della prima linea. È assodato infatti, grazie agli studi di L. Moretti sulle iscrizioni greche di Roma (IGUR) e allo studio di M. L. Lazzarini sui testi ostiensi (Lazzarini 1992-1993, p. 137) che la proporzione delle iscrizioni greche, non solo nell'Urbe, ma anche a Ostia è pari al $3 \%$ rispetto a quelle integra, (h cm 16,9; largh. $\mathrm{cm} 8,7$ in alto e 9,0 in basso; spess. cm 0,1-0,2), reca un'iscrizione su 30 righe, la prima delle quali in latino, tutte le altre in greco $^{15}$. Le linee del testo sono parallele al lato corto ed incise sulla faccia interna della laminetta, mentre il verso è anepigrafe. L'iscrizione si estende per tutta la larghezza della lamina, ma non occupa integralmente l'altezza disponibile e si concentra nei primi $13 \mathrm{~cm}$ dall'alto; la parte inferiore è liscia e priva di lettere o di altri segni. Il testo è inciso, come di consueto, con un oggetto acuminato, ma alcune parole (nelle prime tre righe, in grassetto nel testo) sembrano essere state ripassate con un oggetto a punta più larga e arrotondata. Lo stato di conservazione è ottimo, se si eccettuano alcune minime abrasioni superficiali ed alcune lacune che tuttavia non ne inficiano nel complesso la comprensione. Rimangono forti incertezze sulla lettura della

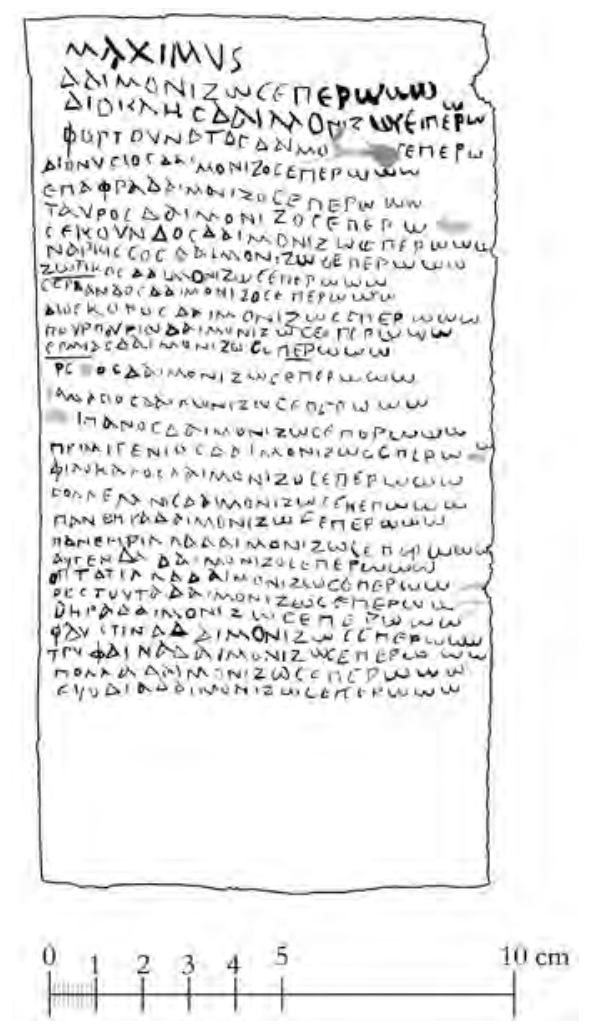

Fig. 8 - Riproduzione grafica della tabella defixionis.

latine. La decisione di adottare la lingua greca potrebbe dunque essere indice di una specifica appartenenza sociale e culturale del committente. Si deve tuttavia ricordare che l'uso del greco nelle defixiones, anche in contesti di lingua latina, è piuttosto diffuso, anche perché la lingua straniera in qualche modo ne garantiva la segretezza nonché l'uso di una consolidata formularietà magica. 
parte iniziale delle 1l. 15-17, fortemente abrase. Le due lacune principali ${ }^{16}$ sembrano essere il risultato di una perforazione con un oggetto appuntito o un chiodo, secondo una consueta pratica magica, anche se forse la superficie della lamina potrebbe essere stata solo battuta e non trapassata completamente ${ }^{17}$.

Soffermandoci ad analizzare la paleografia del documento (fig. 9) si nota che il ductus, tenuto conto del carattere dell'iscrizione e del suo supporto, si presenta piuttosto regolare ed equilibrato, anche se le linee hanno andamento tendenzialmente ondulato e sono in genere inclinate verso il basso a destra. Il testo non è perfettamente allineato a sinistra e le righe hanno lunghezza variabile, il che dipende essenzialmente dal nome del defisso, dato che la formula che lo segue è identica ${ }^{18}$. Lo spazio interlineare è abbastanza costante, mentre non vi è separazione tra le parole, che formano una scriptio continua. Le lettere, con progressiva riduzione dell'altezza (prima riga: $\mathrm{cm} 0,4-0,5$; rimanenti righe $\mathrm{cm} 0,2-0,3$, a parte alcune lettere, come I e $\Phi$, che possono raggiungere un'altezza di cm 0,4-0,5), sono abbastanza irregolari e con tendenza alla grafia corsiva. A con il tratto centrale verticale e il tratto obliquo prolungato; Z, M, N, $\Sigma$ corsivi; $\Sigma, \mathrm{E}, \Omega$ lunati; $\Delta, \Lambda$ con il tratto obliquo prolungato; E con il tratto mediano distaccato; $\mathrm{K}$ con i tratti obliqui distaccati; P con l'occhiello aperto; $\Upsilon$ regolare o privo del tratto verticale. Il testo, tracciato da una sola mano, non è privo di qualche anomalia, come ad esempio i due omega sovrapposti per mancanza di spazio (l. 3) e la legatura tra alcune lettere; talora il lapicida si ispira a tipi dell'epigrafia latina ( $\mathrm{ad}$ esempio nelle $\Upsilon$ rese come $\mathrm{V})$. Infine è opportuno segnalare che, tra la 1.10 e la 1.11 e tra la 1.14 e la 1.15 , nella parte sinistra del documento, è incisa una linea orizzontale lunga $\mathrm{cm}$

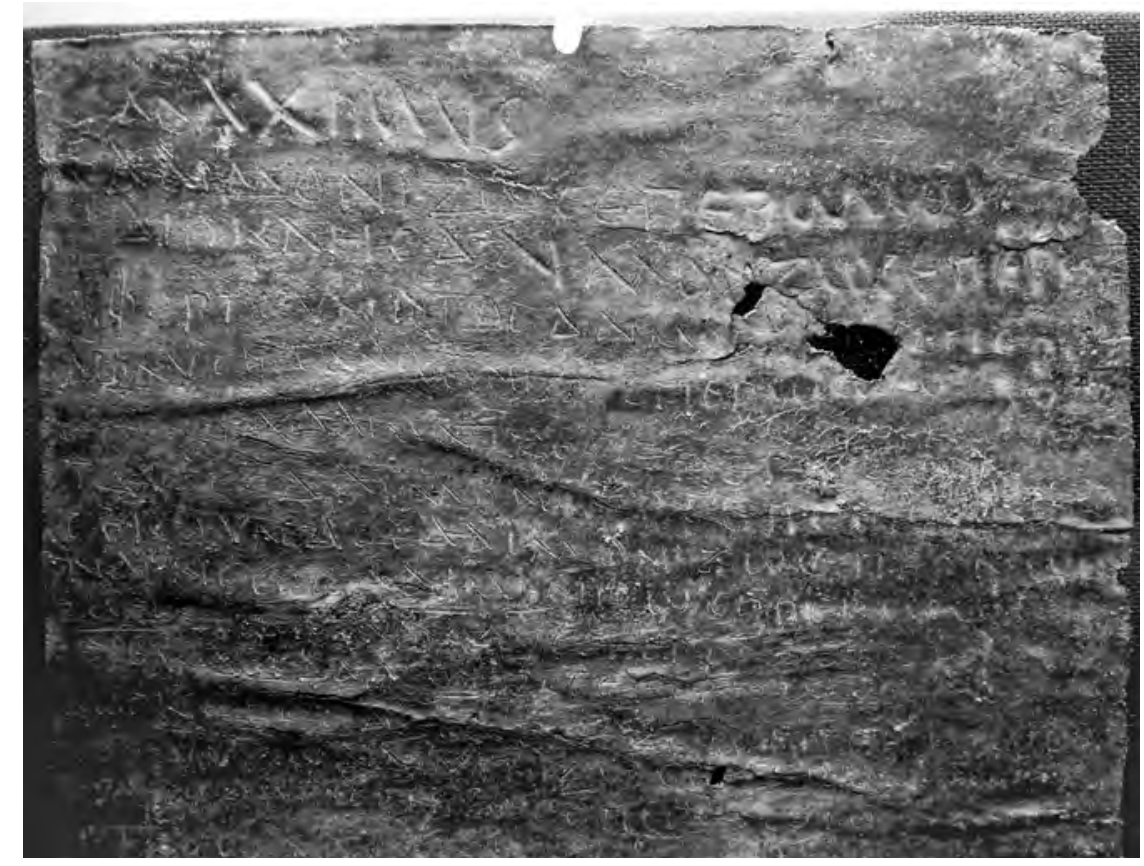

Fig. 9 - Particolare della prima parte della tabella defixionis.

\footnotetext{
${ }^{16}$ Si tratta di due piccoli fori dai margini irregolari ubicati nella parte superiore destra del documento, tra la quarta e sesta riga: il primo a sinistra (largh. cm 0,2, h. cm $0,3)$, il secondo a destra, poco più in basso del precedente (largh. 0,7 , h. 0,4). La lacuna è in questo caso facilmente integrabile in quanto coincide con il tratto del testo in cui ricorre la formula magica ripetuta identica in tutte le righe.
}

${ }^{17} \mathrm{Si}$ veda, ad esempio, una defixio dalla Via Ostiense che presenta una analoga tipologia di fori: Bevilacqua Colacicchi 2006-2007, p. 304, 340-341, fig. 9-10. Cf. anche Cesano 1910, p. 1562.

${ }^{18} \mathrm{Di}$ conseguenza in alcuni rari casi (ll. $\left.3,4,18\right)$ è stato necessario, in presenza di antroponimi molto lunghi, abbreviare la formula finale $\pi \varepsilon \rho \omega \omega \omega$ (eliminando uno o due $\Omega$ ) per mancanza di spazio oppure sovrascrivere un $\Omega(1.3,18)$. 
0,7 che sembra essere stata tracciata deliberatamente e che potrebbe essere intesa come linea di demarcazione tra varie parti del testo ${ }^{19}$, magari funzionale a dividere gruppi di nomi, secondo una logica che tuttavia ci sfugge, oppure costituire una delle consuete sottolineature o soprallineature frequenti nelle defixiones ${ }^{20}$.

Nel testo sono presenti alcune scorrettezze fonetiche e minimi errori (ad esempio l'inversione di omicron e sigma finali del nome Pourpourios), non inconsueti nelle defixiones per via del livello culturale, di norma non molto elevato, di colui che trascriveva il documento. Data l'assenza di un vero e proprio discorso logico, non è possibile riscontrare errori gram-

\section{TRASCRIZIONE}

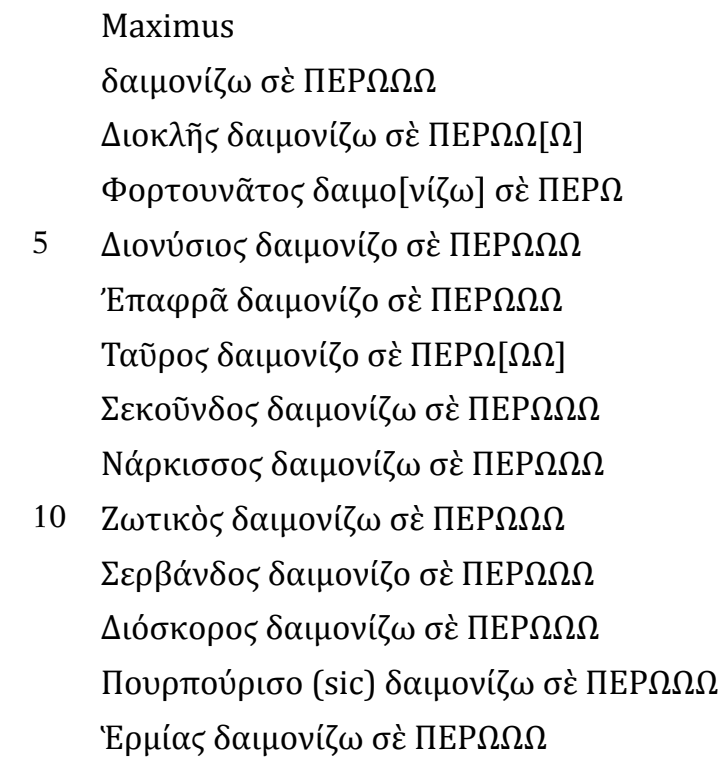

L. 3: il secondo $\omega$ è ascritto al di sopra del primo per assenza di spazio; un terzo $\omega$ è probabilmente da integrare a destra del precedente. L. 4: $\alpha$ e ı sono legati. L. 6: o e $v$ sono legati. L. 10: ı e $\mu$ sono legati. L. 13: o e $\sigma$ finali del nome Поuртоú dell'incisore. L. 15: l'antroponimo è lacunoso (si potrebbe eventualmente integrare Прóßo

\footnotetext{
${ }^{19}$ Bevilacqua 2010, p. 103-106.

${ }^{20}$ Bevilacqua 1998, p. 117; Bevilacqua - Colacicchi 2006-2007, p. 304 (con bibliografia).

${ }^{21}$ La compresenza nelle epigrafi di termini tratti dalla
}

maticali e sintattici, altrimenti frequenti in questo genere di testi. Alcune inesattezze o peculiarità nella trascrizione degli antroponimi sono dovute, probabilmente, alla traslitterazione in greco di nomi latini ${ }^{21}$ : lo scrivente, pur utilizzando l'alfabeto greco, probabilmente pensava e pronunciava i nomi in latino (vedi ad esempio 'Е $\alpha \varphi \rho \tilde{\alpha}$ in luogo della forma greca

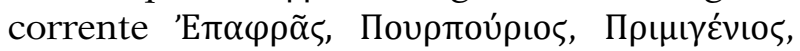

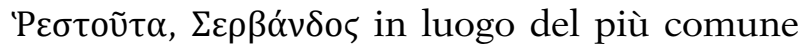
$\Sigma \varepsilon \rho o u \alpha ́ v \delta o \zeta)$. Anche il fatto che in sei casi la $\omega$ finale di $\delta \alpha \mu o v i \zeta \omega$ sia resa con o indica l'uso quotidiano di un alfabeto, quello latino, in cui entrambi i suoni erano scritti allo stesso modo.

15

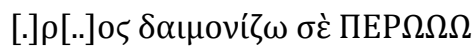

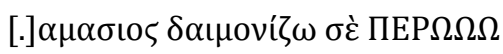

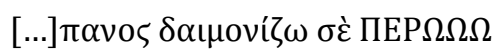

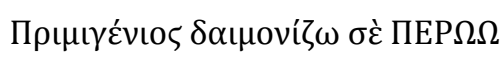

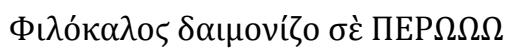

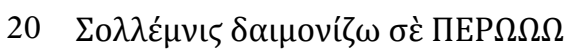

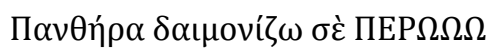

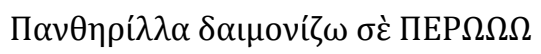

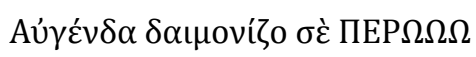

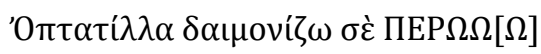

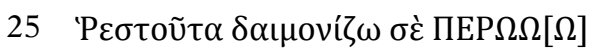

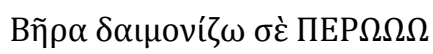

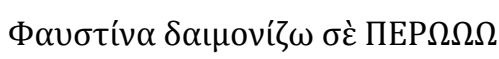

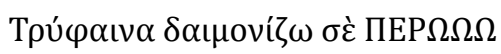

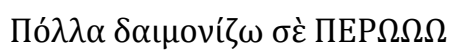

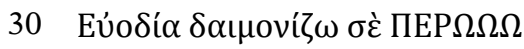

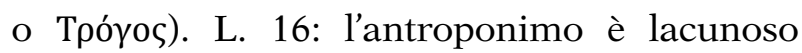

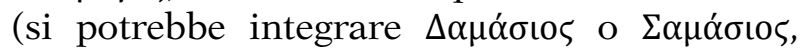
entrambi rari e non attestati in questa forma, ovvero, espungendo il segno iniziale, leggere

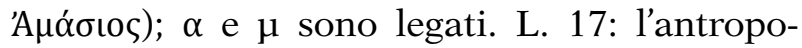
nimo è lacunoso (si potrebbe forse integrare

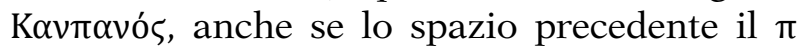

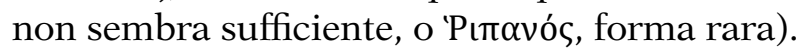

lingua greca e da quella latina è stata, in maniera molto calzante, definita «bilinguismo imperfetto»: Kramer 1984; cf. Bevilacqua 1998, p. 128-129. 
Pur nella consapevolezza che, soprattutto in testi come le defixiones, i criteri paleografici sono sempre assai rischiosi ai fini di una definizione cronologica puntuale, è opportuno evidenziare che i caratteri del testo in esame sono piuttosto accurati e, pur essendo in parte corsiveggianti, sono ancora ben scritti ed abbastanza chiari. I caratteri latini della prima riga appartengono alla capitale corsiva e trovano confronti con documenti della piena età imperiale: si segnala la $\mathrm{A}$ con il tratto centrale verticale e disarticolato, la $\mathrm{M}$ semplice con tratti divaricati, la V obliqua ${ }^{22}$. Lo stesso vale per il testo greco, nel quale si notano le seguenti peculiarità: la $\mathrm{H}$ non è ancora priva del tratto verticale destro in alto, la $\mathrm{M}$ è aperta, la $\mathrm{B}$ ha i tratti non connessi, la E è lunata e si chiude in basso, la $\Upsilon$, quando priva del tratto verticale, è ancora simmetrica e non con il tratto destro aperto. L'ambito cronologico a cui si può attribuire il testo, quindi, anche sulla base del confronto con gli elementi desumibili dal contesto archeologico, è la piena età imperiale, più precisamente tra gli ultimi decenni del II e i primi decenni del III sec. d.C.

Dal punto di vista del contenuto, si tratta di un testo piuttosto semplice consistente in una lista di nomi propri al nominativo ${ }^{23}$, seguiti dalla formula di defissione. Il nome del defigens, come nella maggior parte delle defixiones note, non compare per ovvie ragioni di riservatezza. I defissi sono tutti indifferentemente indicati con un simplex nomen, senza il patronimico per i Greci e con il solo cognome per i Romani. Il primo nome della lista (Maximus), evidentemente quello della persona più importante o del coordinatore o capo del gruppo dei defissi, è espresso in latino ed è inciso in posizione isolata in alto a sinistra, con caratteri di dimensioni maggiori rispetto alle linee successive: la formula di defissione che segue $(\delta \alpha \iota \mu$ oví $\zeta \omega \sigma \varepsilon)$ è

\footnotetext{
${ }^{22} \mathrm{Si}$ vedano in merito, tra gli altri, Bartoletti 1990, p. 43 e la tavola comparativa di Fox (Fox 1912, pl. VIII).

${ }^{23}$ Per le liste nominali di defissi, con specifiche caratteristiche formali (impaginazione rigida e paratassi) che le rendevano ben confacenti alle pratiche magiche, cf. in particolare: Audollent 1904 passim; Panciera 1968, p. 335-338; Solin 1968, p. 13; Scarfi 1972; Jordan 1985b, p. 172, n. 82; p. 176 , n. 105 ; p. 177 , n. 110 ; Bevilacqua 2010, p. 89 e 95-97.

${ }^{24} \mathrm{Vi}$ è incertezza sul numero effettivo degli uomini e delle donne in quanto due degli antroponimi, Sollemnis
}

invece in greco. Di seguito sono elencati i nomi di altre 28 persone oggetto dell'astio del defigens, prima gli uomini e poi le donne ${ }^{24}$, accompagnati tutti dalla medesima formula di maledizione. La scelta dell'uso del nominativo per indicare i destinatari della maledizione è dettata da esigenze di chiarezza e non è infrequente nelle defixiones, anche quando la grammatica richiederebbe un altro caso. Peraltro, al fine di evitare confusioni di persona, si prestava particolare attenzione alla trascrizione grafica esatta dei nomi: tale norma è quasi sempre rispettata nella defixio, ad eccezione di isolati casi in cui gli antroponimi presentano piccole imprecisioni.

Il fatto che i personaggi siano individuati da un unico nome lascia presupporre un ambiente sociale piuttosto basso, verosimilmente di origine servile o eventualmente libertina: nel caso in cui vi fossero dei liberti, comunque, distinguerli dagli schiavi è impossibile, mancando l'aggiunta del gentilizio al cognome. I nomi latini e quelli di origine greca grosso modo si equivalgono numericamente e sono elencati senza alcun apparente ordine o logica; sono invece distinti, come si è già accennato, i nomi femminili, che si trovano alla fine del documento e tra i quali prevalgono antroponimi romani.

La grande varietà onomastica costituisce un'ulteriore conferma del panorama multietnico portuense nella piena età imperiale. Il rinvenimento dell'iscrizione in una città portuale renderebbe infatti plausibile l'origine dal mondo greco e dal Mediterraneo orientale di alcuni dei personaggi elencati, ma bisogna anche in questo senso essere cauti ${ }^{25}$. È opportuno formulare qualche considerazione sull'onomastica dei personaggi: se infatti alcuni degli antroponimi attestati sono estremamente comuni e non necessitano di discussione né di analisi più approfondite, altri invece sono più rari e pertanto è su di essi che è opportuno

e Panthera, posti proprio nel punto di discrimine tra uomini e donne nella lista, possono essere sia maschili che femminili. Abbiamo quindi un numero di uomini che può variare da 18 a 20 e conseguentemente le donne sarebbero in numero variabile tra 11 e 9.

${ }^{25}$ La cautela è d'uopo dal momento che, come è noto, non sempre l'onomastica straniera è da riferirsi ad un'effettiva provenienza geografica, quanto piuttosto si fonda sovente su mode o su scelte dei patroni (Scarfi 1972, p. 66). 
soffermarsi. Da segnalare innanzi tutto che non vi sono omonimi né nomi che tradiscano una sicura origine geografica.

Tra i nomi di origine greca più diffusi, attestati variamente nell'area di Roma, Ostia e Portus dalla tarda età repubblicana fino al IV

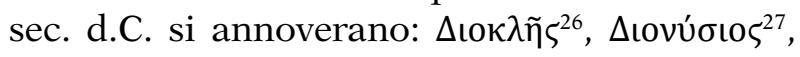

${ }^{26}$ Per le attestazioni in latino cf. Solin 2003, p. 42-43. Ad Ostia un Diocles è il dedicante di un'epigrafe nel Mitreo di Lucrezio Menandro (Bloch 1953, p. 244, n. 8); un altro Diocles vi è attestato in un'epigrafe inedita. Nella necropoli dell'Isola Sacra il nome è presente una sola volta (Helttula 2007, p. 155-156, n. 133).

${ }^{27}$ Si tratta di un nome diffusissimo e ve ne sono numerose attestazioni a Roma ed Ostia con diverse varianti grafiche, dal I sec. a.C. al V sec. d.C. (Solin 2003, p. 323-329). A Portus e nella necropoli dell'Isola Sacra il nome, in greco, ricorre in un'epigrafe funeraria non databile con esattezza (Sacco 1984, p. 70, n. 50) e, in latino, una volta nella forma maschile (Thylander 1952, p. 286-287, B103) ed una in quella femminile (Helttula 2007, p. 248-249, n. 232).

${ }^{28}$ Il nome Nó so dal tardo I sec. a.C. fino al IV sec. d.C. sia in iscrizioni latine (Solin 2003, p. 1183-1185) che in iscrizioni greche. Per attestazioni a Portus e nella necropoli dell'Isola Sacra, cf. Thylander 1952, p. 271, B72; Helttula 2007, p. 126-127, n. 109 (C. Terentius Narcissus).

${ }^{29}$ Zoticus è assai diffuso a Roma (Solin 2003, p. 895897) e a Ostia, soprattutto in età imperiale. Nella necropoli dell'Isola Sacra è noto un Ti. Iulius Zoticus da due epigrafi (Helttula 2007, p. 316-317, n. 312 e p. 319-320, n. 315) di età adrianeo-antonina. A Portus invece il nome è attestato tre volte (Thylander 1952, p. 249-250, B32; p. 407-408, B343.2; p. 408-418, B344.254).

${ }^{30}$ L'antroponimo è ampiamente diffuso a Roma, tra il I sec. a.C. e il IV sec. d.C., (Solin 2003, p. 364-366). Ad Ostia non è molto frequente: se ne conoscono almeno cinque attestazioni latine, databili negli ultimi anni del II o nei primi decenni del III sec. d.C. (CIL, XIV, 1108; 4569, V 29; 4562, 2, a12; 4562, 4, II13; Bloch 1953, p. 291-292, n. 54). A Portus invece è attestato una sola volta, nel corpus fabrum navalium (CIL, XIV, 256 = Thylander 1952, p. 408418, B344, 160).

31 L'antroponimo è ampiamente attestato a Roma (Solin 2003, p. 811-813). Ad Ostia ricorre in due epigrafi provenienti dalla cd. Curia, l'una del 171-300 d.C., l'altra del 208-218 d.C., entrambe contenenti liste di duoviri quinquennales (CIL, XIV, 4562, 4; 4563, 1). A Portus è noto un Claudius Philocalus nel corpus fabrum navalium (Thylander 1952, p. 408-418, B344, 150).

${ }^{32}$ L'antroponimo è attestato a Roma e ad Ostia dal I al V sec. d.C. in diverse varianti grafiche (ad esempio Euhodia, Euodia, Eubodia, Heuhodia, Euuodia etc.): cf. Solin 2003, p. 1311-1312. A Portus e nella necropoli dell'Isola sacra conosciamo una Euhodia (Helttula 2007, p. 317-318, n. 313) ed un Eubodius in un'epigrafe cristiana (Thylander 1952, p. 343-344, B236).

${ }^{33}$ Il nome, in greco, in questa forma è relativamente

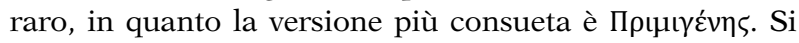

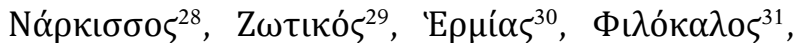
Eúo $\delta \alpha^{32}$. Tra gli antroponimi più comuni di origine latina, traslitterati tutti in greco, tranne

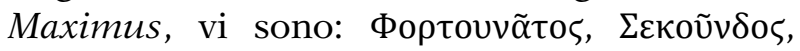

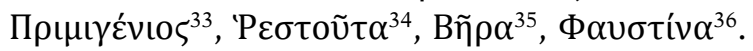

Meno diffusi sono alcuni nomi greci quali

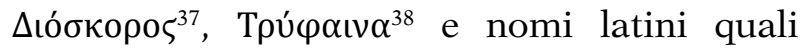

tratta di un cognomen diffusissimo (Kajanto 1965, p. 290). Nella necropoli dell'Isola Sacra sono noti, tra le altre attestazioni del nome, due liberti di nome Primigenius, entrambi vissuti in età traianea-adrianea (per il primo, cf. $C I L, \mathrm{XIV}, 1030=$ Helttula 2007, p. 229, n. 202; il secondo è il dedicatario di un'epigrafe funeraria in situ nel 1930 sulla tomba 50, databile sempre in età traianea: Helttula 2007, p. 63-64, n. 51).

${ }^{34}$ Il nome latino Restutus/Restuta, dal quale l'antroponimo in esame è traslitterato, è abbastanza diffuso e costituisce una abbreviazione di Restitutus/Restituta. Nella forma greca il nome è attestato in una defixio dall'anfiteatro di Cartagine del II sec. d.C. (Audollent 1904, p. 339-341, n. 249). Il

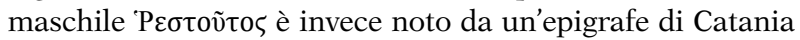
della seconda metà del IV sec. d.C. (IG, XIV, 529). Per quanto concerne la versione latina, nella necropoli dell'Isola Sacra, esso ricorre in una nota epigrafe funeraria del II sec. d.C. in memoria di una donna gettata nel Tevere dal marito recante i nomi dei due genitori, Restutus Piscinesis e Prima Restuta (Helttula 2007, p. 325-326, n. 321); a Portus un Fabius Restutus è menzionato nell'elenco del corpus fabrum navalium (Thylander 1952, p. 408-418, B344, 49) ed una Roscia Restuta in un'epigrafe funeraria (Thylander 1952, p. 348, B243). Anche ad Ostia il nome ricorre diverse volte.

${ }^{35}$ Il cognomen è molto diffuso in età romana imperiale (Kajanto 1965, p. 253). Nella necropoli dell'Isola Sacra

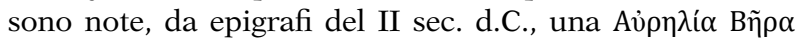
(Sacco 1984, p. 52-53, n. 32) ed una Septueia Vera (Helttula 2007, p. 327-328, n. 324).

${ }^{36}$ Cognomen assai diffuso, sia al maschile che, soprattutto, al femminile (Kajanto 1965, p. 272), in particolare a partire dal II sec. d.C. Nella necropoli dell'Isola Sacra sono note una Cl(audia) Faustina (Helttula 2007, p. 13, n. 12) ed una Ofillia Faustina (Helttula 2007, p. 231, n. 208). Anche a Portus il cognomen è attestato tre volte (Thylander 1952, p. 286-287, B103; p. 344, B237; p. 347-348, n. B242).

${ }^{37} \mathrm{Il}$ nome, non particolarmente comune, è attestato a Roma in diverse epigrafi latine nel periodo compreso tra il I e il III sec. d.C. (Solin 2003, p. 511); in greco ricorre ad esempio in IGUR, I, 191 e 193 (dediche a Serapide del tardo III sec. d.C.) e in IGUR I, 130 (dedica alla Magna Mater del 244 d.C.). Ad Ostia è anche abbastanza raro e lo si trova, ad esempio, in due brevi epigrafi funerarie (CIL, XIV, 909; 1439).

${ }^{38}$ Diffuso in tutto l'impero, in particolare in Asia Minore, il nome è ampiamente attestato a Roma (Solin 2003, p. 847-848; IGUR, II, 509). Nella necropoli dell'Isola Sacra si conosce una Genucia Tryphaena (Helttula 2007, p. 18-19, n. 16), mentre ad Ostia sono note una Volumnia Tryphaena (AE 1964, 219a), una Aufidia Tryphaena (AE 1982, 133), una Caesia Tryphaena (CIL, XIV, 734), una Egrilia Tryfaenia (CIL, XIV, 998). 


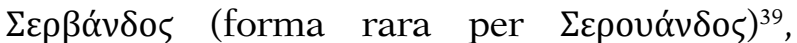

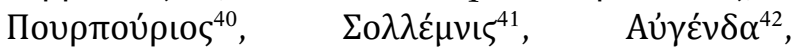
'0 Taurus, i quali, pur se di origine certamente greca-orientale, hanno un corrispondente altrettanto diffuso nell'onomastica latina ${ }^{44}$. Meno frequenti, ma sempre piuttosto noti,

${ }^{39}$ La lettura del nome sembra certa, pur se della versione greca, in questa grafia, se ne conoscono rarissime attestazioni, tra cui si ricorda un testo del V sec. d.C. da Tomis (IScM, II, 390). Meno infrequente invece la versione

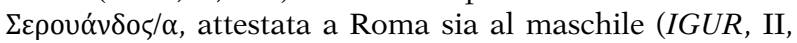
889) che al femminile (IGUR, II, 1042). Il corrispettivo latino Servandus è ampiamente noto (Kajanto 1965, p. 360). Nella necropoli dell'Isola Sacra si conosce una Varia P(ubli) $f($ iliae) Servanda grazie a due epigrafi quasi identiche poste da due dei suoi liberti all'ingresso del recinto e al di sopra della porta della tomba $n$. 87 , databili nella prima metà del II sec. d.C.; un altro testo più breve dedicato alla medesima persona era posto all'interno della tomba stessa al di sotto della nicchia centrale della parete di fondo (da ultima, Helttula 2007, p. 123-126, nn. 106-108). Ad Ostia ricordiamo, tra le rare attestazioni di questo antroponimo, $C I L, \mathrm{XIV}, 1345$.

${ }^{40} \mathrm{Si}$ tratta della traslitterazione in greco del nome latino Purpurio/Purpurius, non attestata altrove in questa forma: nei testi greci ricorre esclusivamente nella versione

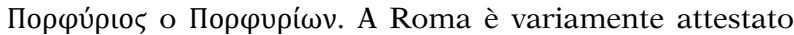
(Kajanto 1965, p. 230). Ad Ostia un Tiberius Herennius Purpurius è menzionato in un elenco di corporati databile durante il regno di Gallieno (CIL, XIV, 5357; Marinucci 1992, p. 190-192, C47, II9).

${ }^{41}$ Sollemnis (Kajanto 1965, p. 221), nome prevalentemente maschile e in un unico caso anche femminile, ricorre in epigrafi funerarie da Roma e suburbio nel I-III sec. d.C. Ad Ostia un Sollemnis è menzionato in un'epigrafe proveniente dal Mitreo di Porta Romana (CIL, XIV, 4571, a10). Il nome, abbastanza raro nella forma greca, è attestato ad esempio a Doura Europos in un testo del 231 d.C. (Little-Rowell 1933, p. 72-74, n. 169; SEG, 7, 333) in cui si menziona il consularis Klaoudios Sollemnios Pakatianos, noto anche in un'epigrafe latina dall'Arabia (IGLS, XIII,1 9076) ed in un testo dal foro romano del 232-235 d.C. (CIL, VI, 41181).

${ }^{42}$ L'antroponimo Augendus/Augenda è noto raramente e solo al maschile in iscrizioni greche, tra cui si segnala un'epigrafe funeraria a Roma (IGUR, II, 283: Aî̀ı

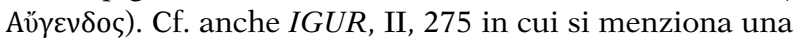

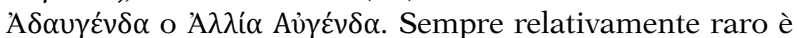
il latino Augenda: a Roma è attestato in un'epigrafe funeraria (Gatti 1926, p. 247, n. 18, Statilia Augenda), mentre la versione maschile Augendus è più diffusa (Kajanto 1965, p. 360). Non se ne conoscono al momento attestazioni, né al maschile né al femminile, a Portus, Isola Sacra ed Ostia.

${ }^{43}$ Il diminutivo di Optata non è noto al momento in epigrafi greche e se ne hanno alcune rare attestazioni latine: sembra che il cognomen fosse più diffuso al femminile che non al maschile (Kajanto 1965, p. 296). Tra di esse sono invece altri antroponimi, quali 'E $\pi \alpha \varphi \rho \tilde{\alpha} \zeta-$ Epaphra $^{45}$ e Пó $\lambda \lambda \alpha^{46}$, assai diffusi a Roma e dei quali si conoscono alcune attestazioni latine ad Ostia, ma nessuna a Portus. Abbastanza desueti anche i nomi П $\alpha v \theta \eta \dot{\eta} \rho \alpha^{47}$ e, soprattutto, l'ipocoristico $\Pi \alpha v \theta \eta \rho i ́ \lambda \lambda \alpha^{48}$. Il primo può essere sia maschile che femminile e, nella lista, si trova

ricordiamo un'epigrafe da Roma (CIL, VI, 13364: Aurelia Optatilla), una dall'area di Mediolanum (CIL, V, 5658: Calpurnia L(uci) f(ilia) Optatilla), una a Filippi (Pilhofer 2009, p. 175, n. 151). Cf. anche una defixio da Siscia con un elenco di nomi, tra cui un' 'О $\pi \tau \tau \tilde{\alpha} \tau \alpha: S E G, 46,1380$ = Curbera - Jordan 1996. In Numidia è invece noto un M(arcus) C[- -] Optatillus (ILAlg II, 2376) da un'epigrafe funeraria databile nel II sec. d.C. L'antroponimo Optata è attestato nella necropoli dell'Isola Sacra (Helttula 2007, p. 78-79, n. 65), mentre il maschile Optatus vi ricorre tre volte (Thylander 1952, p. 401-402, B337; p. 408-418, B344, 176; p. 430-431, B378).

${ }^{44}$ L'antroponimo è assai diffuso a Roma e ad Ostia (Kajanto 1965, p. 329). A Portus un Taurio è noto da un'epigrafe funeraria della prima metà del II sec. d.C. (Helttula 2007, p. 73-74, n. 61).

${ }^{45}$ A Roma ne sono note numerose attestazioni, databili dal I sec. a.C. fino al III sec. d.C. (Solin 2003, p. 349-351; IGUR, I, 160 A44; IGUR, II, 435; IGUR, III, 1358). Ad Ostia lo si ritrova in alcune epigrafi latine, in diverse varianti grafiche, tra le quali preme ricordare: Barbieri 1982-1983, p. 149-150, n. 93 (Epaphra); Bloch 1953, p. 278-282, n. 42, dVIII.1 (Epafra); CIL, XIV, 1305 (Epaphra); CIL, XIV, 595 (Epaphra); CIL, XIV, 1052 (Aepafra).

${ }^{46}$ Polla (Paula), corrispettivo femminile del cognomen Paulus, inizialmente diffuso come praenomen, diventa un cognomen frequentemente attestato, anche poi in età cristiana per motivi chiaramente comprensibili (Kajanto 1965 , p. 243-244). Non se ne hanno attestazioni a Portus e solo due sono i casi sinora accertati ad Ostia (Barbieri 1958, p. 152-153; CIL, XIV, 598).

${ }^{47}$ La versione femminile a Roma è nota in tre documenti risalenti alla seconda metà del II o agli inizi del III sec. d.C. (Solin 2003, p. 1145): si tratta di una Memmia Panthera (CIL, VI 28593), di una Iulia Panthera (Ferrua

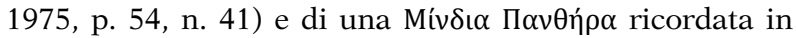
un'epigrafe funeraria del III sec. d.C. (CIL, VI, $22520=$ IGUR, II, 793). Nella forma maschile Panthera è attestato a Roma in totale quattro volte dall'epoca di Augusto al II sec. d.C. (Solin 2003, p. 1146). Ad Ostia nell'iscrizione latina su un'urna cineraria dedicata a madre e figlia, la figlia si chiama Geminia Pantheris (CIL, XIV, 4930), ma vi è attestato anche Panthera in un documento ancora inedito. Non abbiamo testimonianze del nome a Portus.

${ }^{48}$ L'antroponimo non è attestato in epigrafi greche e, nella forma latina, è noto da un'unica iscrizione su un'urna cineraria rinvenuta sulla via Portuense (zona Pozzo Pantaleo), dedicata ad una schiava dal coniuge Dionysius e databile durante il regno di Antonino Pio: Paribeni 1922, p. 411, n. 4; Solin 2003, p. 1146. 
nel punto di discrimine tra i nomi degli uomini e quelli delle donne (peraltro anche il nome che lo precede, $\Sigma o \lambda \lambda \varepsilon \dot{\mu \nu} \iota \varsigma$, generalmente maschile, può essere, come si è visto, anche femminile).

Purtroppo, in mancanza di un'onomastica più complessa e di ulteriori indizi intrinseci al testo, ogni speranza di chiarire l'identità dei personaggi menzionati risulta vana. Alcuni antroponimi ricorrono, come si è visto, in altre iscrizioni portuensi o ostiensi, ma non vi è alcun elemento indicativo dell'eventuale corrispondenza o coincidenza dei personaggi citati nella defixio con quelli in esse menzionati, né si è in grado di stabilire eventuali relazioni di tipo parentale o di altra natura tra gruppi di nomi tramite il confronto con le altre epigrafi. Data l'estrema stringatezza del testo, inoltre, è impossibile definire quali fossero le funzioni dei personaggi menzionati, né sono chiari gli elementi che li accomunavano e li rendevano parte di un unico gruppo, del quale Maximus doveva essere il capo ovvero il personaggio più influente, che in qualche maniera ne garantiva l'identificazione agli occhi del defigens. Il nome Maximus, data la sua ampia diffusione, non permette in alcun modo di tentare di comprendere l'identità del personaggio tramite eventuali confronti.

Il numero elevato di defissi è una caratteristica relativamente rara, che trova confronti 1968)

${ }^{49}$ La defixio latina elenca circa 30 persone (Solin

${ }^{50} \mathrm{Nel}$ caso della defixio latina di Altino si tratta di un elenco di 23 persone (ma il numero potrebbe salire a 27 se si desse una diversa interpretazione del testo). Cf. Scarfi 1972, p. 60.

${ }^{51}$ InscrIt, X, 1, 592.

${ }^{52}$ Si tratta di una lista di 29 nomi, sia greci che latini, di cui 19 maschili ed 8 femminili, databile nel III sec. d.C. (Curbera - Jordan 1996).

53 Audollent 1904, p. 149-151, n. 96 e p. 153-154, n. 101 .

${ }^{54}$ Alla tomba 95 (in prossimità della quale, come si è detto, si trovava la sepoltura che ha restituito la defixio) è stata attribuita, pur se in via ipotetica, l'epigrafe dedicata in età adrianea da $M$. Antonius Vitalis, membro del collegio dei cultores Larum Portus Augusti. Si veda in merito Thylander 1952, p. 34-35, A19; Helltula 2007, p. 147; 267268, n. 253; cf. Lazzarini 1991, p. 71, nt. 173. A titolo di mera suggestione, si potrebbe valutare l'ipotesi che, se i ad esempio ad Ostia ${ }^{49}$, ad Altino ${ }^{50}$, a Pola ${ }^{51}$, a Siscia ${ }^{52}$ ed in Germania ${ }^{53}$. Anche questo elemento deve essere tenuto in considerazione al fine di tentare di comprendere chi fossero i 29 defissi. Potrebbe trattarsi dei servi di una familia, oppure dei membri di un'impresa commerciale legata al porto o, meno verosimilmente, di un'azienda agricola o di un collegio. La presenza di numerose donne sembra tuttavia indurre a propendere per l'ipotesi che si tratti dei servi/liberti di una familia o comunque di una comunità privata di altro genere ${ }^{54}$.

Nel testo non si invoca esplicitamente alcuna divinità né si utilizzano le consuete formule di defissione: l'unico elemento indicativo della punizione che si vuole infliggere ai nemici è il verbo generico $\delta \alpha \mu \nu$ oví $\zeta \omega$, ripetuto dopo ciascun nome e seguito dal pronome personale $\sigma \varepsilon ́$. Tale verbo non rientra nel formulario consueto attestato in altre defissioni e non è attestato epigraficamente, se si eccettua un brevissimo testo cristiano da Didyma ${ }^{55}$.

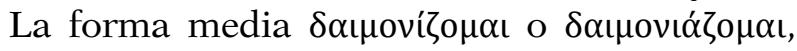
equivalente a $\delta \alpha \iota \mu \nu \iota^{\alpha} \omega^{56}$ implica «l'essere posseduto da un demone» ed è attestata in due Papiri Magici ${ }^{57}$ ma non in altre defixiones. Al medio il verbo ricorre in Plutarco ${ }^{58} \mathrm{e}$, soprattutto, in diverse fonti cristiane, in particolare nei Vangeli di Matteo, Marco, Giovanni ${ }^{59}$, dove assume la valenza di «essere posseduto

defissi erano parte di un collegio, potessero forse appartenere proprio al collegium menzionato nell'iscrizione affissa sulla tomba monumentale.

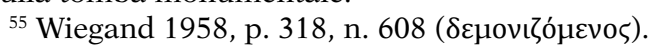

${ }^{56}$ Liddell - Scott 1996, p. 365.

${ }^{57}$ Nei papiri il verbo, al participio, individua le persone possedute dal demonio. PGM, IV 3008; Betz 1986,

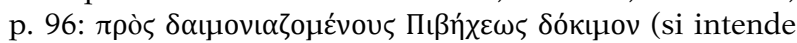
qui dare una pozione, di cui si espone di seguito la ricetta, per coloro che sono posseduti dal demone). Il termine è attestato anche in un papiro di Antinoupolis del VI sec. d.C. (PAnt, II, 66, 1. 17 = Suppl. Mag., II, p. 211-221, n. 94, 1. 17)

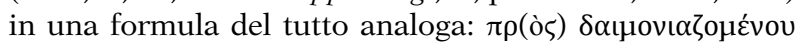
[ 5 c. 9]. Per una recente messa a punto sulle formule di scongiuro nei Papiri Magici Greci, vedi Zografou 2015.

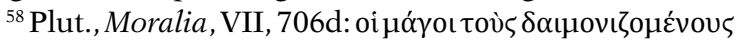

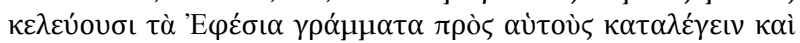
ỏvouó̧̉ıเv.

${ }^{59}$ Ev. Matt. 4, 24; 8, 16; 8, 28; 8, 33; 9, 32; 12, 22; 15, 22. Ev. Marc. 1, 32; 5, 15; 5, 16. Ev. Johan. 10, 21. 
da uno spirito maligno». In un'epigrafe oracolare da Termessos, in Pisidia, si menziona la

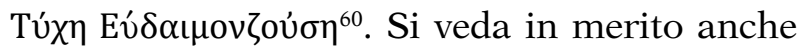
il Lessico di Esichio che riporta la seguente

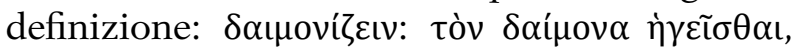

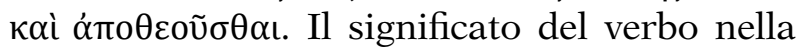
forma attiva, desumibile anche dalla radice semantica, dovrebbe essere dunque «affidare ai demoni», «demonizzare». Si può inoltre fare riferimento a perifrasi di analogo valore utiliz-

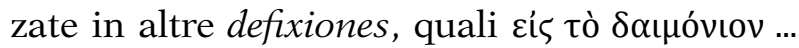

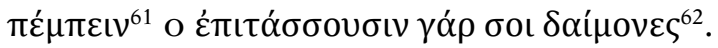

Come è noto i $\delta \alpha i ́ \mu o v \varepsilon \varsigma$-daemones, in cui si devono riconoscere le anime dei defunti, erano spesso considerati capaci di recepire e trasmettere le maledizioni alle divinità infere e ad essi ci si rivolgeva collocando le laminette arrotolate o ripiegate all'interno delle tombe ${ }^{63}$. Il

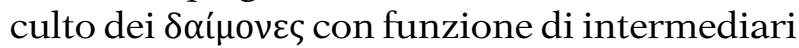
tra gli dei e gli uomini si diffonde in età imperiale e rientra in una nuova corrente filosoficoteologica sviluppatasi a partire dal II secolo d.C. È quindi naturale che i demoni vengano spesso menzionati nelle defixiones e in altre iscrizione magiche, sia in quelle greche che in quelle latine. In merito al rapporto dei daemones con gli uomini vale la pena ricordare anche una lamina di piombo, forse dal Fayum, in cui il richiedente chiede al demone di entrare nel suo corpo e di parlare attraverso la sua bocca ${ }^{64}$.

Non chiaramente interpretabili sono le lettere $(\pi \varepsilon \rho \omega \omega \omega)$ che seguono la formula di maledizione e si leggono chiaramente alla fine di ciascuna riga. Si può pensare ad una sorta

${ }^{60}$ TAM, III, 1, 34, A 29.

${ }^{61}$ Woodward - Austin 1925-1926, p. 73-74, n. 13; Jordan 1985b, p. 165-166, n. 54.

62 L'espressione - «che i demoni ti comandino»ricorre in una defixio da Cartagine del II-III sec. d.C. (Audollent 1904, p. 316-318, n. 238, 1l. 18-19).

${ }^{63}$ Bravo 1987, in part. p. 208-211 (per l'accezione del termine daimon in epoca ellenistica); Gager 1992; Bravo 1995, p. 521 e 524-525; Ciraolo 1995, p. 284.

${ }^{64}$ Suppl. Mag., II, p. 76-80, n. 66. Cf. Minutoli 2015, p. 61. di formula magica priva di un reale significato, che non trova diretti confronti in altri testi noti o, eventualmente, ad una serie di $\chi \alpha \rho \alpha \kappa \tau \tilde{\eta} \rho \varepsilon \zeta^{65}$. Nel tentativo di ricondurre $\pi \varepsilon \rho \omega \omega \omega$ ad un vocabolo di senso compiuto, escludendo le due omega finali (interpretabili come $\chi \alpha \rho \alpha \kappa \tau \tilde{\eta} \rho \varepsilon \zeta ?)$, si potrebbe pensare al verbo $\pi \varepsilon \rho \alpha ́ \omega$, nel significato di "attraversare, trafiggere, penetrare», con riferimento alla pratica magica di trafiggere il defisso ${ }^{66}$. Anche in questo caso però non mi sono noti confronti diretti dell'uso di questo termine in altre defixiones. La formula $\pi \varepsilon \rho \omega$ è attestata un'unica volta in un papiro magico ${ }^{67}$.

Non sono specificate le cause della maledizione, la cui menzione sarebbe stata particolarmente utile per tentare di individuare il contesto di appartenenza del gruppo di defissi: non è da escludere che il defigens facesse parte del medesimo gruppo e pertanto volesse vendicarsi con gli altri membri di eventuali torti subiti. Non è quindi possibile definire con esattezza a quale delle categorie canoniche delle defixiones sia da attribuire il testo, pur se rimane verosimile che esso rientri tra quelli dettati da generiche ragioni di odio e non in una classe più specifica. Si potrebbero supporre, alla base dell'odio per i personaggi elencati, dispute ed invidie di tipo economico o legate ad attività imprenditoriali e commerciali, il che troverebbe facilmente spiegazione in un contesto come quello di Ostia e Portus. In conclusione forse l'ipotesi più verosimile rimane quella che la maledizione sia indirizzata ad una familia legata ad un dominus, di cui però nulla di più possiamo dire, ma alla quale forse apparteneva anche il defigens.

${ }^{65}$ Una ripetizione di charakteres, tra cui diverse $\omega$, si ha ad esempio in una defixio dalla Fonte di Anna Perenna a Roma, databile nel IV sec. d.C. Vedi Blänsdorf 2010, p. 221 e 235-236.

${ }^{66}$ Liddell - Scott 1996, p. 1365. Meno plausibile l'ipotesi di ricondurre le lettere in questione al verbo $\pi \eta \rho$ ó $\omega$ (presupponendo un'errata trascrizione della $\eta$ in $\varepsilon$ ) che significa «mutilare, castrare». Cf. Liddell - Scott 1996, p. 1401-1402.

${ }^{67}$ PGM IV, 1149. 
BIBLIOGRAFIA

IGLS XIII = M. Sartre, Inscriptions grecques et latines de la Syrie, XIII, 1, Bostra, Parigi, 1982.

$I G U R \mathrm{I}=\mathrm{L}$. Moretti, Inscriptiones Graecae Urbis Romae, fasciculus primus, Roma, 1968.

IGUR II = L. Moretti, Inscriptiones Graecae Urbis Romae, fasciculus secundus, Roma, 1972-1973.

IGUR III = L. Moretti, Inscriptiones Graecae Urbis Romae, Fasciculus Tertius, Roma, 1979.

ILAlg II = S. Gsell (a cura di H.-G. Pflaum), Inscriptions latines de l'Algérie. II. Inscriptions de la confédération cirtéenne, de Cuicul et de la tribu des Suburbures, Parigi, 1957.

InscrIt XIII, 1 = B. Forlati Tamaro, Inscriptiones Italiae, X, Regio X, I, Pola et Nesactium, Roma, 1947.

IScM II = I. Stoian, Inscriptiones Scythiae Minoris Graecae et Latinae. II. Tomis et Territorium, Bucureşti, 1987.

PAnt II = J. W. B. Barns, H. Zilliacus, The Antinoopolis Papyri, Part II, Londra, 1960.

$P G M=$ K. Preisendanz, Papyri Graecae Magicae. Die Griechischen Zauberpapyri, I, Leipzig-Berlino, 1928.

Suppl. Mag. II = R. W. Daniel, F. Maltomini (a cura di), Supplementum Magicum. I-II, Opladen, 1990-1992 (Papyrologica Coloniensia, XVI).

TAM III = R. Heberdey, Tituli Asiae Minoris. III. Tituli Pisidiae, Vindobona, 1941.

Audollent 1904 = A. Audollent, Defixionum tabellae quotquot innotuerunt tam in Graecis Orientis quam in totius Occidentis partibus praeter Atticas in Corpore Inscriptionum Atticarum editas, Parigi, 1904.

Barbieri 1958 = G. Barbieri, Le iscrizioni delle necropoli, in Floriani Squarciapino 1958, p. 129165.

Barbieri 1982-1983 = G. Barbieri, La collezione epigrafica Iaia, in BCom, 88, 1982-1983, p. 105-189.

Bartoletti $1990=\mathrm{G}$. Bartoletti, La scrittura romana nelle tabellae defixionum (secc. I a.C. $-I V$ d.C.). Note paleografiche, in ScrCiv, 14, 1990, p. 7-47.

Betz 1986 = H. D. Betz, The Greek Magical Papyri in Translation, Including the Demotic Spells, Chicago, 1986.

Bevilacqua 1998 = G. Bevilacqua, Due nuove defixiones greche da Roma, in Epigraphica, 60, 1998, p. 113-134.

Bevilacqua 2010 = G. Bevilacqua, Scrittura e magia . Un repertorio di oggetti iscritti della magia grecoromana, Roma, 2010 (Opuscula Epigraphica, 12).
Bevilacqua - Colacicchi 2006-2007 = G. Bevilacqua, O. Colacicchi, Roma. Una nuova defixio latina dalla via Ostiense, in NSc, 17-18, 2006-2007, p. 303-349.

Blänsdorf $2010=\mathrm{J}$. Blänsdorf, The Texts from the Fons Annae Perennae, in R. L. Gordon e F. M. Simón (a cura di), Magical Practice in the Latin West. Papers from the International Conference held at the University of Zaragoza. 30 Sept.-1 Oct. 2005, Leida-Londra, 2010 (Religions in the Graeco-Roman World, 168), p. 215-244.

Bloch 1953 = H. Bloch, Ostia. Iscrizioni rinvenute tra il 1930 e il 1939, in NSc, 7, 1953, p. 239-306.

Bravo 1987 = B. Bravo, Une tablette magique d'Olbia Pontique, les morts, les héros et les démons, in Poikilia. Études offertes à Jean-Pierre Vernant, Parigi, 1987, p. 185-218.

Bravo 1995 = B. Bravo, Magia tra virgolette? Sull'Antologia di defixiones pubblicata da J. G. Gager, in Athenaeum, 73, 1995, p. 517-525.

Calza 1940 = G. Calza, La necropoli del Porto di Roma nell'Isola Sacra, Roma, 1940.

Cesano 1910 = L. Cesano, Defixio, in E. De Ruggiero, Dizionario epigrafico di Antichità romane, C-E, II, 2, Spoleto, 1910, p. 1558-1591.

Ciraolo 1995 = L. J. Ciraolo, Supernatural Assistants in the Greek Magical Papyri, in M. Meyer, P. Mirecki (a cura di), Ancient Magic and Ritual Power, Leida-New York-Cologna, 1995, p. 279295.

Curbera - Jordan 1996 = J. B. Curbera, D. R. Jordan, A Curse Tablet from Pannonia Superior, in Tyche, 11, 1996, p. 45-50.

Ferrua 1975 = A. Ferrua, Via Paisiello e Panfilo, in $R A C r, 51,1975$, p. 27-61.

Floriani Squarciapino $1958=$ M. Floriani Squarciapino (a cura di), Scavi di Ostia, III. Le necropoli. I. Le tombe di età repubblicana $e$ augustea, Roma, 1958.

Fox 1912 = W. S. Fox, The Johns Hopkins Tabellae Defixionum, Baltimore, 1912 (Suppl AJPh, 33.1)

Gager 1992 = J. G. Gager, Curse Tablets and Binding Spells from the Ancient World, New York-Oxford, 1992.

Gatti 1926 = E. Gatti, Notizie di recenti trovamenti di antichità in Roma e nel suburbio, in NSc, 54, 1926, p. 235-269

Graf 1994 = F. Graf, La magie dans l'Antiquité grécoromaine. Idéologie et pratique, Parigi, 1994.

Helttula 2007 = A. Helttula (a cura di), Le iscrizioni sepolcrali latine nell'Isola Sacra, Roma, 2007 (Acta Istituti Romani Finlandiae, 30). 
Jordan 1985a = D. R. Jordan, Defixiones from a Well near the Southwest Corner of the Athenian agora, in Hesperia, 54, 1985, p. 205-255.

Jordan 1985b = D. R. Jordan, A Survey of Greek Defixiones Not Included in the Special Corpora, in GrRomByzSt, 26, 1985, p. 151-197.

Kajanto 1965 = I. Kajanto, The Latin Cognomina, Helsinki, 1965 (Commentationes humanarum litterarum, 36, 2).

Kramer $1984=$ J. Kramer, Testi greci scritti nell'alfabeto latino e testi latini scritti nell'alfabeto greco: un caso di bilinguismo imperfetto, in Atti del XVII Congresso internazionale di papirologia, III, Napoli, 1984, p. 1377-1384.

Lazzarini $1991=$ S. Lazzarini, Sepulcra familiaria. Un'indagine epigrafico-giuridica, Padova, 1991.

Lazzarini 1992-1993 = M. L. Lazzarini, $I$ Greci di Ostia, in ScAnt, 6-7, 1992-1993, p. 137-141.

Liddell - Scott $1996=$ H. G. Liddell, R. Scott, $A$ Greek-English Lexicon, Oxford, 1996.

Little-Rowell 1933 = A. McN. G. Little, H. T. Rowell, IV. Greek inscriptions, in P. V. C. Baur, M. I. Rostovtzeff, A. R. Bellinger (a cura di), The Excavations at DuraEuropos. Preliminary Report of Fourth Season of Work. October 1930-March 1931, Londra, 1933, p. 72-79.

Marinucci 1992 = A. Marinucci, Iscrizioni, in P. Cicerchia, A. Marinucci (a cura di), Scavi di Ostia, XI. Le Terme del Foro o di Gavio Massimo, Roma, 1992, p. 165-228.

Minutoli 2015 = D. Minutoli, Exempla divari supporti scrittori contenenti testi magici provenienti da Antinoupolis, in M. de Haro Sanchez (a cura di), Écrire la magie dans l'Antiquité. Actes du colloque international (Liège, 13-15 octobre 2011), Liegi, 2015, p. 51-67.

Mura 1996 = M. I. Mura, Le tabellae defixionum africane come fonte di storia sociale: nota preliminare, in L'Africa romana. Atti dell'XI
Convegno di Studio, Cartagine, 15-18 dicembre 1994, Ozieri, 1996, p. 1535-1546.

Panciera 1968 = S. Panciera, Due novità epigrafiche romane. 2. Una defixio latina del Palatino, in RendLinc, 23, 1968, p. 332-340.

Paribeni $1922=$ R. Paribeni, Via Portuense. Rinvenimenti di tombe di età imperiale, in NSc, 19, 1922, p. 408-427.

Piccaluga $2010=$ G. Piccaluga, Tecnica grafica $e$ liturgia magica nelle tabellae defixionum, in StMatStoRel, 76, 1, 2010, p. 13-19.

Pilhofer 2009 = P. Pilhofer, Philippi. Band II. Katalog der Inschriften von Philippi, Tübingen, 2009.

Sacco 1984 = G. Sacco, Iscrizioni greche d'Italia . Porto, Roma, 1984.

Scarfi 1972 = B. M. Scarfi, Una tabella defixionis $d a$ Altino (Venezia), in Epigraphica, 34, 1972, p. 55-68.

Solin 1968 = H. Solin, Eine neue Fluchtafel aus Ostia, in Commentations humanarum litterarum, 42, 3, 1968, p. 3-31.

Solin $2003=$ H. Solin, Die griechischen Personennamen in Rom. Ein Namenbuch. Zweite, völlig neu bearbeitete Auflage, Berlino-New York, 2003.

Thylander $1952=\mathrm{H}$. Thylander, Inscriptions du port d'Ostie, Lund, 1952 (Acta Instituti Romani regni Sueciae, 4, 1-2).

Wiegand $1958=$ T. Wiegand, Didyma. II. Die Inschriften, Berlino, 1958.

Woodward - Austin 1925 = A. M. Woodward, R. P. Austin, Some Note-Books of Sir William Gell, in BSA, 27, 1925-1926, p. 67-80.

Youtie - Bonner $1937=$ H. C. Youtie, C. Bonner, Two Curse Tablets from Beisan, in TransactAmPhilAss, 68,1937 , p. $43-77$ e 128.

Zografou 2015 = A. Zografou, Les formules d'adjuration dans les Papyrus Grecs Magiques, in M. de Haro Sanchez (a cura di), Écrire la magie dans l'Antiquité. Actes du colloque international (Liège, 13-15 octobre 2011), Liegi, 2015, p. 267-280. 


\section{SOMMARIO}

Mireille Cébeillac-Gervasoni et Fausto Zevi, Premessa

Lista delle abbreviazioni

\section{LA SPONDA DESTRA DEL TEVERE, PRESSO LA FOCE, PRIMA DEI ROMANI}

Francesca Romana De CAstro, Alessandra Facciolo, Monica Gala, Maria Cristina Grossi, Cinzia Morelli, Maria Lucrezia Rinaldi, Daria Ruggeri e Sandra Sivilli, La sponda destra del Tevere, presso la foce, prima dei Romani: gli insediamenti

Valeria Acconcia, Francesca Romana De Castro e Chiara Morciano, I materiali

\section{LA NECROPOLI DI PORTO ALL'ISOLA SACRA: VECCHIE E NUOVE RICERCHE}

Ida Baldassarre, Irene Bragantini, Anna Maria Dolciotti, Chiara Morselli e Franca Taglietti, Necropoli dell'Isola Sacra. Le ricerche 1968-89: ripercorrendo un'esperienza

Paola Olivanti e Marcello Spanu, Necropoli dell'Isola Sacra, scavo 1988-1989: alcune riflessioni su occupazione degli spazi, cronologia delle sepolture, corredi

Luciano Camilli e Franca Taglietti, Sepolture e monete: il prezzo dell'Ade? A proposito dei rinvenimenti monetali in tombe della necropoli di Porto all'Isola Sacra

Luciano Camilli e Franca Tagliettr, Contributi per un'archeologia di cantiere: i bolli laterizi dalla necropoli di Porto all'Isola Sacra

Emanuela Borgia, Una tabella defixionis dalla necropoli dell'Isola Sacra

Luca Bondioli, Paola Germoni e Paola Francesca Rossi, L'infante e il sarcofago delle Muse dall'Isola Sacra

Paola Germoni, Simon Keay, Martin Millett e Kristian Strutt, Ostia beyond the Tiber: recent archaeological discoveries in the Isola Sacra

Patrizia TuRI, Lo scavo preventivo in Via della Basilica di Sant'Ippolito (anno 2012): un'area sepolcrale periferica 


\section{IL SETTORE SUD-ORIENTALE DI OSTIA}

Simona Pannuzi, Viabilità e utilizzo del territorio. Il suburbio sud-orientale di Ostia alla luce dei recenti rinvenimenti archeologici

Paola GERMONI, Indagini preventive nel suburbio meridionale di Ostia

Ascanio D'andrea, Lucia De Gregorio, Paola Germoni e Carla Ninel Pischedda, Indagini archeologiche preventive nel settore sudorientale extraurbano di Ostia Antica: nuove acquisizioni sulla via litoranea e la linea di costa antica

Paola Germoni, Lucia De Gregorio, Carla Ninel Pischedda e Ascanio D'Andrea, Indagini archeologiche preventive nell'area della necropoli di Pianabella (area 12): nuove acquisizioni per la ricostruzione del paesaggio extraurbano di Ostia Antica tra I e IV secolo d.C.

Mireille Cébeillac-Gervasoni, Maria Mimmo e Matthias Bruno, Il materiale epigrafico dall'area 12 ...

Luca Bondioli, Alessia Nava, Paola Francesca Rossi e Alessandra Sperduti, Lo studio antropologico delle sepolture di Larcius Felix e Onesime

\section{VARIA: ARCHEOLOGIA ED EPIGRAFIA}

Massimiliano DAvID, Il nuovo mitreo dei marmi colorati sulla via della Marciana a Ostia Antica ......

Luke Lavan, Chronology in Late Antiquity: a lesson from the Palaestra

Roberta Geremia Nucci, Un Capricorno ad Ostia (anzi due)

Fausto Zevi, Programma U.I.G. (Una Iscrizione al Giorno): iscrizioni funerarie di Ostia

Filippo Marini Recchia, Un'iscrizione inedita di Agrippa e altre nuove ricongiunzioni epigrafiche ostiensi

Nicolas LaUBRY, Iura sepulcrorum à Ostie : un supplément ...

Christer BRUUn, Celebrazioni ad Ostia: la scelta del giorno per le dediche pubbliche, le inaugurazioni e altri eventi collettivi

Françoise van HAEPEREN, Quelques réflexions sur la fonction des Fastes d'Ostie

Indice delle fonti

Indice dei luoghi

Riassunti

Sommario 

Achevé d'imprimer

en juin 2019

sur les presses de

Estilo Estugraf

Impresores, S.L.

Ciempozuelos

(Madrid) Espagne 